\title{
Identification of a cluster-situated activator of oxytetracycline biosynthesis and manipulation of its expression for improved oxytetracycline production in Streptomyces rimosus
}

\author{
Shouliang Yin ${ }^{1+}$, Weishan Wang ${ }^{2 \dagger}$, Xuefeng Wang ${ }^{3}$, Yaxin Zhu², Xiaole $\mathrm{Jia}^{3}$, Shanshan Li ${ }^{2}$, Fang Yuan ${ }^{3}$,
} Yuxiu Zhang ${ }^{1 *}$ and Keqian Yang ${ }^{2 *}$

\begin{abstract}
Background: Oxytetracycline (OTC) is a broad-spectrum antibiotic commercially produced by Streptomyces rimosus. Despite its importance, little is known about the regulation of OTC biosynthesis, which hampered any effort to improve OTC production via engineering regulatory genes.

Results: A gene encoding a Streptomyces antibiotic regulatory protein (SARP) was discovered immediately adjacent to the otrB gene of oxy cluster in S. rimosus and designated otcR. Deletion and complementation of otcR abolished or restored OTC production, respectively, indicating that otcR encodes an essential activator of OTC biosynthesis. Then, the predicted consensus SARP-binding sequences were extracted from the promoter regions of oxy cluster. Transcriptional analysis in a heterologous GFP reporter system demonstrated that OtcR directly activated the transcription of five oxy promoters in E. coli, further mutational analysis of a SARP-binding sequence of oxyl promoter proved that OtcR directly interacted with the consensus repeats. Therefore, otc $R$ was chosen as an engineering target, OTC production was significantly increased by overexpression of otcR as tandem copies each under the control of strong SF14 promoter.

Conclusions: A SARP activator, OtcR, was identified in oxy cluster of S. rimosus; it was shown to directly activate five promoters from oxy cluster. Overexpression of otc $R$ at an appropriate level dramatically increased OTC production by 6.49 times compared to the parental strain, thus demonstrating the great potential of manipulating OtcR to improve the yield of OTC production.
\end{abstract}

Keywords: Oxytetracycline, Activator, SARP, Rational engineering, Streptomyces rimosus

\section{Background}

Oxytetracycline (5'-hydroxytetracycline; OTC) and related tetracyclines are potent inhibitors of bacterial protein synthesis with broad-spectrum activity against both grampositive and gram-negative pathogens [1]. Although the clinical use of the tetracyclines has declined in recent

\footnotetext{
*Correspondence: zhangyuxiu@cumtb.edu.cn; yangkq@im.ac.cn

${ }^{\dagger}$ Equal contributors

'Department of Environmental and Biological Engineering, School of Chemical and Environmental Engineering, China University of Mining and Technology (Beijing), D11 Xueyuan Road, Haidian District, Beijing 100083, People's Republic of China

${ }^{2}$ State Key Laboratory of Microbial Resources, Institute of Microbiology, Chinese Academy of Sciences, 1 Beichen West Road, Chaoyang District, Beijing 100101, People's Republic of China

Full list of author information is available at the end of the article
}

years due to their side effects and reduced efficacy, OTC had found wide application in animal feeds and aquaculture, with annual production exceeding 5,000 ton.

OTC is an aromatic polyketide antibiotic commercially produced by Streptomyces rimosus. The OTC biosynthetic gene cluster is located in a $34 \mathrm{~kb}$ segment of $S$. rimosus genome flanked by two putative resistance genes (otrA and otrB) [2]. Initially, several genes from the cluster were partially characterized, i.e. genes encoding minimal PKS (otcY1-1, otcY1-2, and otcY1-3) [3], cyclase $(o t c D 1)$ [4], and anhydrotetracycline oxygenase $($ otcC) $[5,6]$. However, the complete sequence of the OTC biosynthetic gene cluster was not released to the public, so in 2006, Tang's group systematically re-sequenced the 
OTC biosynthetic gene cluster and re-named the cluster oxy [7]. Afterward, Tang's group further characterized OTC biosynthetic pathway, by studying several key biosynthetic enzymes, such as the amidotransferase OxyD and the thiolase OxyP for the synthesis of the malonamate starter unit $[7,8]$, the ancillary oxygenase OxyE for more efficient C-4 hydroxylation [9] and two redox enzymes OxyS and OxyR for final transformation of anhydrotetracycline to OTC [10]. However, for the regulation of OTC biosynthesis, there is a paucity of curated information, only one annotated regulatory gene otrR was reported which is divergently transcribed from the resistance gene $\operatorname{tr} B$ [11]. OtrR is a MarR family regulator, but the role of OtrR in OTC production had not been investigated [11]. Based on its location, it could be involved in the regulation of $\operatorname{otr} B$, which encodes a MFS family efflux pump probably involved in the export of OTC [11].

Over the years, further clues on the regulation of OTC biosynthesis had emerged. McDowall et al. [12] reported that the sequences in the promoter regions of otcC, otcX, and otc $Y$ contain tandem repeats, that are similar to the DNA-binding sites of Streptomyces antibiotic regulatory proteins (SARP) transcription activators, with this information they proposed that OTC production could be controlled by an unknown SARP-like activator. Besides, when expressing the OTC cluster in the heterologous host Streptomyces coelicolor CH999, Wang et al. [13] found that the SARP regulator, encoded by $c t c 11(c t c B)$ from the chlorotetracycline (CTC) gene cluster $(c t c)$ of Streptomyces aureofaciens [14], could activate the transcription of $o x y$ cluster in heterologous host. Since OTC and CTC are structurally similar antibiotics, they should share similar biosynthetic and regulatory mechanisms. The fact that a SARP regulator (Ctc11) exists in the CTC cluster and Ctc11 could activate oxy cluster in the heterologous host strongly implies that a SARP-like regulator may exist in the vicinity of oxy cluster. Therefore, in this work, our goal is to identify the SARP-like activator of oxy cluster and engineer it to improve OTC production.

Engineering the regulation of antibiotic biosynthesis is an effective strategy to improve antibiotic production [15]. Simple overexpression of pathway-specific activator gene using a constitutive promoter was often used to improve antibiotic production [16]. This simple genetic engineering has been successful in some cases $[16,17]$, but can fall short to optimize the expression of target gene to get the best production [18]. In the era of synthetic biology, fine-tuning gene expression to achieve optimal level of target production has been accepted as key to successful engineering practice $[18,19]$. Therefore, as a prelude to an engineering attempt, in this work, a series of experiments were performed to determine the optimal expression level of the newly identified activator (OtcR) to give the best yield improvement.

In this work, a cluster-situated SARP-like regulator, OtcR, was discovered immediately adjacent to the resistance gene $\operatorname{otr} B$ in the upstream of $o x y$ cluster. OtcR was confirmed to be a pathway specific activator of OTC biosynthesis, and overexpression of OtcR at an appropriate level significantly increased the production of OTC.

\section{Results}

\section{Discovery of SARP regulator OtcR}

The SARP regulator Ctc11 from the $c t c$ gene cluster of $S$. aureofaciens was found to activate the expression of oxy cluster in heterologous host S. coelicolor CH999 [13], which strongly suggests that the expression of oxy cluster in S. rimosus is also positively regulated by a native SARP regulator. To find the native SARP regulator of $o x y$ cluster in S. rimosus, a fosmid library of S. rimosus was constructed. First, neighboring genes, up- and down-stream of oxy cluster were targeted and sequenced. As predicted, a SARP regulator was identified immediately upstream of $\operatorname{otr} B$, and designated otcR (Additional file 1: Figure S1A). The complete DNA sequence of otcR has been deposited in GenBank under the accession number KP035101. The amino acid sequence of OtcR is closely related to Ctc11 (sharing 46\% identities, Additional file 1: Figure S1B). They both have an $\mathrm{N}$ terminal DNA-binding domain and a $\mathrm{C}$ terminal bacterial transcriptional activation domain (BTAD), but lack the other extra domains usually found in members of the SARP regulators, such as an ATPase domain or tetratricopeptide repeat (TPR) domain [20,21].

\section{OtcR is essential for OTC production}

To determine the role of $o t c R$ in OTC biosynthesis, an $o t c R$ disruption mutant was constructed via double-crossover recombination (Figure 1A). The correct construction of the mutant $(\triangle \mathrm{otcR})$ was confirmed by PCR (Figure $1 \mathrm{~B})$. Then $\Delta$ otcR was cultured in FM medium to see its effect on OTC production. As expected, in comparison with parental strain S. rimosus M4018, OTC production was completely abolished in $\Delta$ otcR (Figure 1C). Complementation of $\Delta$ otcR with a single copy of otcR driven by its own promoter $(\Delta$ otcR::otcR) restored the production of OTC to a similar level to the parental strain (Figure 1C). To exclude the possibility of growth effect on OTC production, the biomasses of S. rimosus M4018, $\Delta$ otcR, and $\Delta$ otcR::otcR strains were determined simultaneously, the results showed that the three strains grew similarly (Additional file 2: Figure S2), so the positive effect on OTC production was completely attributable to the function of otcR.

\section{Consensus sequences of the oxy promoter regions}

Previously, McDowall et al. observed the tandem repeats recognized by SARP-like regulator in the $o t c C$, otc $X$, and 


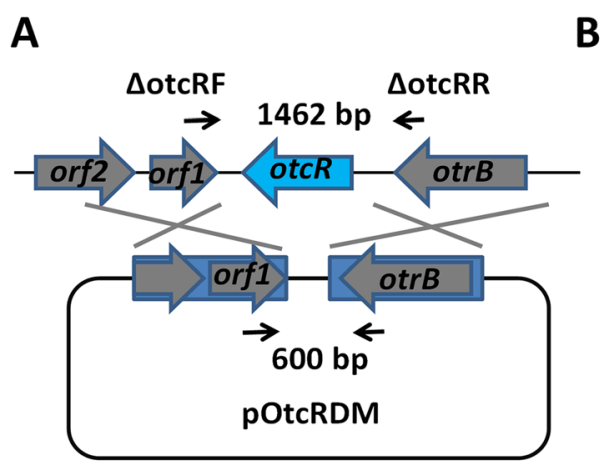

C

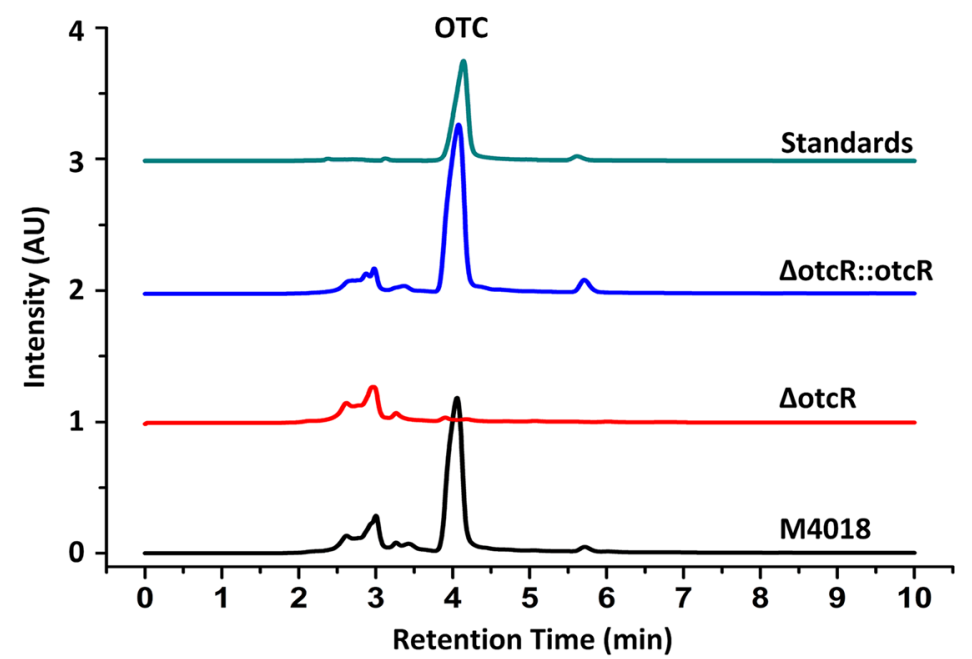

Figure 1 OtcR is essential for OTC production. A. Schematic representation of the strategy used for the disruption of otcR in S. rimosus M4018. The solid arrows indicate the positions of primers used for the confirmation of the disruption mutants ( $\Delta \mathrm{OtcR})$. B. PCR verification of the $\Delta$ otcR mutant. Line M: marker; line 1: PCR products (1462 bp) using the genome of S. rimosus M4018 as template; line 2: PCR products (600 bp) using the genome of $\Delta$ otcR as template. C. OTC production profiles of S. rimosus M4018, $\Delta$ otcR and $\Delta$ otcR::otcR were analyzed by HPLC (UV $350 \mathrm{~nm}$ ).

otc $Y$ promoter regions [12]. To examine the presence of SARP-binding tandem repeats in oxy promoters, the potential consensus sequence motif of oxy promoter regions was extracted; the promoter regions of $c t c$ were simultaneously analyzed because the SARP regulator Ctc11 could activate the expression of oxy cluster. The sequences of $c t c$ and oxy were obtained from GenBank (accession numbers HM627755 [22] and DQ143963 [7], respectively, Figure 2A). As the divergent intergenic regions in both clusters should contain bidirectional promoters, the sequences of these regions were aligned. As shown in Figure 2B, two or three direct hexameric repeats were identified in the promoter regions of $c t c W$, $c t c N, c t c H, o x y I, o x y J, o x y R$, oxyS and oxyA (Figure 2B). Then the consensus sequence of these repeats was calculated by MeMe [23], which displayed the same two hexameric repeats as the results of alignment (Figure 2C). It is important to note that the identified repeats are separated by $11 \mathrm{bp}$, corresponding to one complete turn of DNA helix. It is a typical feature of the binding sites of SARP regulators, as SARPs normally form homodimers in vivo and are expected to bind two direct repeats at the same side of DNA [21].

To further define the consensus SARP-binding motif of the oxy promoters, the promoter regions of oxy (oxyI, $o x y J$, oxyR, oxyS and oxyA) were analyzed by MeMe separately. To our surprise, the consensus motif of oxy showed a little difference from that of $c t c$ cluster, which included two $9 \mathrm{nt}$ direct repeats (Figure 2D). The forward $9 \mathrm{nt}$ repeat is also separated from the adjacent one by $11 \mathrm{bp}$. It should be noted that the 9 nt direct repeats specific for oxy promoters encompass the 6 nt repeats identified from both $o x y$ and $c t c$ cluster.

\section{OtcR directly activates oxy promoters that contain the consensus repeat sequences}

To determine whether OtcR directly interacts with the promoter regions of $\operatorname{oxy} A, \operatorname{oxyI}, \operatorname{oxy} J, \operatorname{oxy} R$ and $\operatorname{oxy} S$, a 


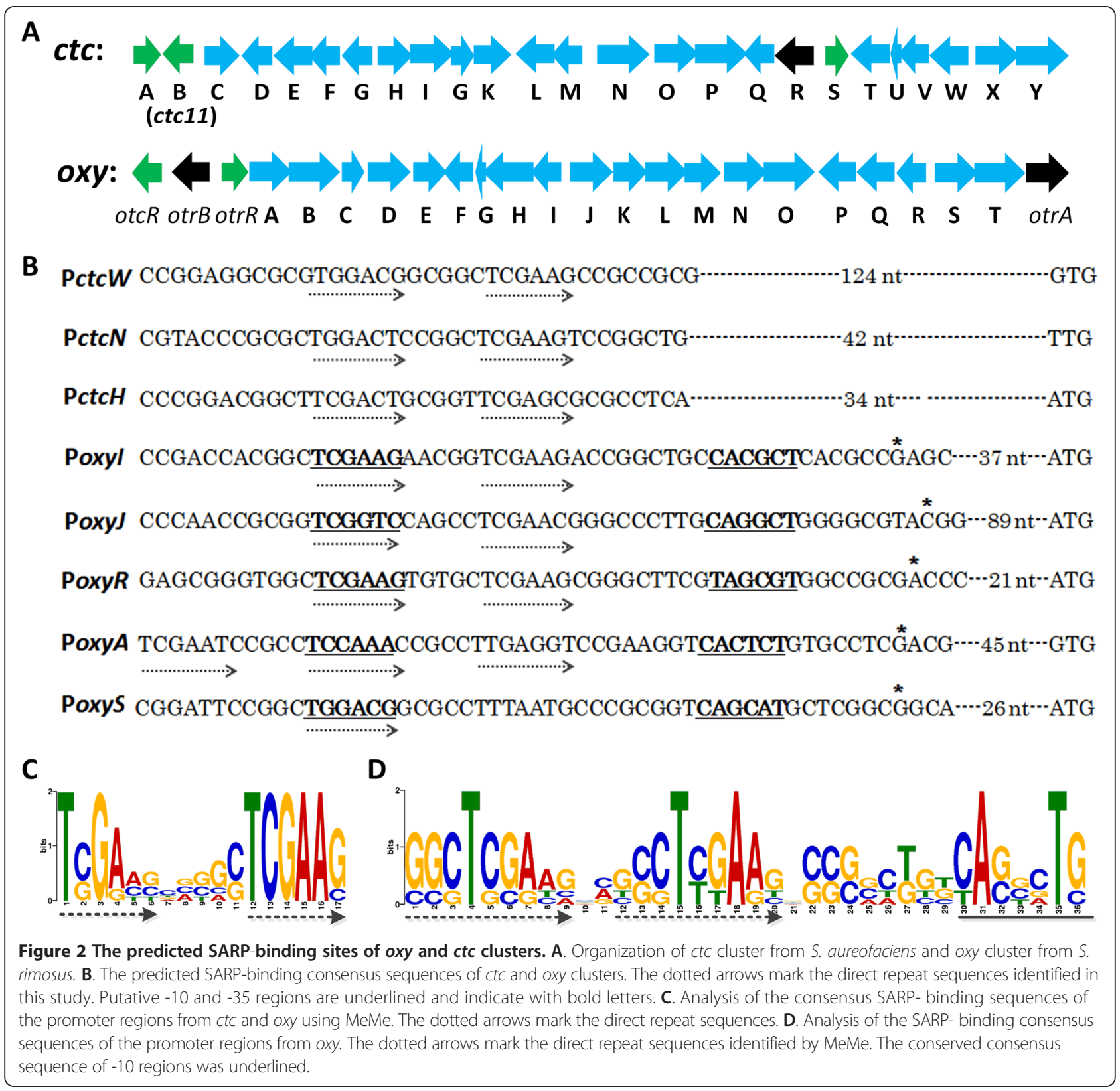

straight forward experiment to perform would be electrophoretic mobility shift assays (EMSA) of OtcR with these regions in vitro. However, soluble expression of recombinant OtcR in $E$. coli failed despite considerable effort. Therefore, we designed a reporter system in $E$. coli to demonstrate the regulatory relationship. As shown in Figure 3A, plasmids, in which $g f p$ gene was directly controlled by promoters of oxyA, oxyI, oxyJ, oxyR and oxyS respectively, were transformed into $E$. coli. The resulting strains were endowed with the abilities to emit green fluorescence (Figure 3B). When otcR driven by SF14 promoter was inserted into the above plasmids (Figure 3A) and transformed into $E$. coli, fluorescences were enhanced ranging from 1.3 to 4 folds compared to that without otcR (Figure 3B). These results indicated that OtcR indeed activates the expression of oxy promoters in a heterologous $E$. coli host.

To further verify whether the regulatory relationship is due to the interaction of OtcR with the 9 nt direct repeats, we mutated the second 9 nt direct repeats (CGGTCGAAG) of oxyI promoter (PoxyI) to CGGTCGTAG (PoxyI1*) and CGGAGGTAG (PoxyI2\%), respectively (Figure 3C), and tested the effects of OtcR on the two mutant promoters using above reporter system. When OtcR was not expressed, the two mutant promoters showed similar activities as PoxyI (Figure 3D). However, when otcR 
A

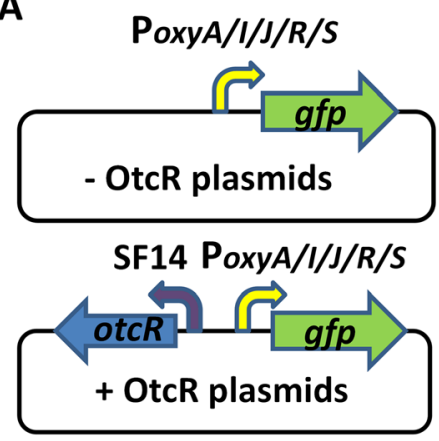

C

Poxyl:
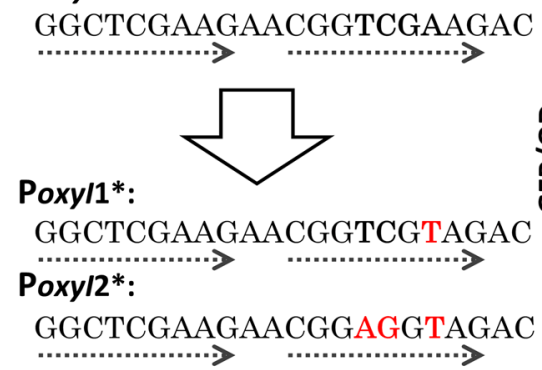

B

D
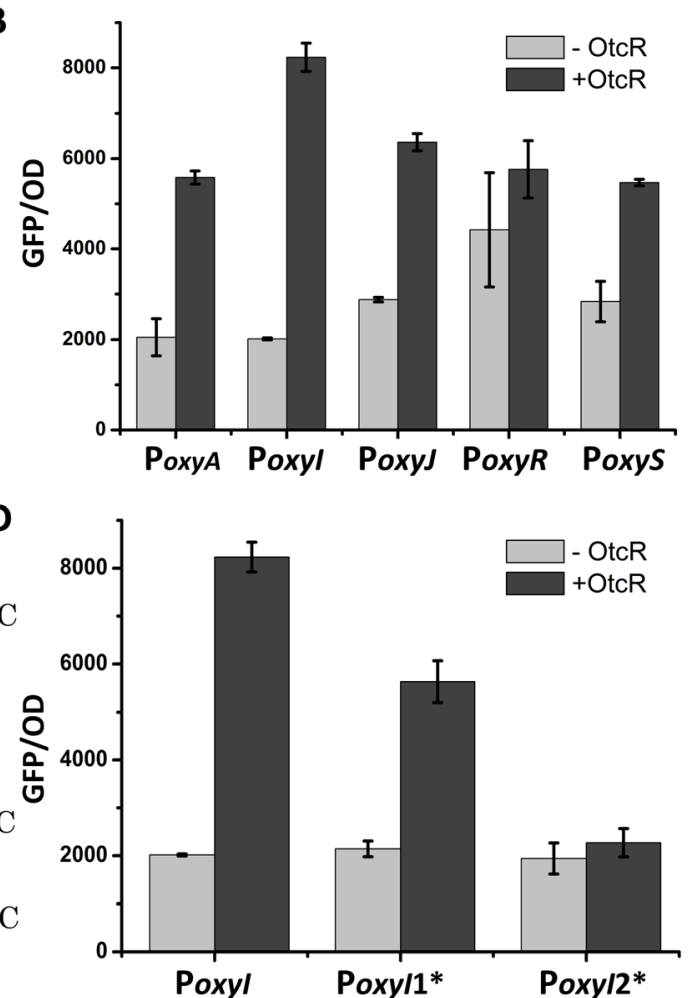

Figure 3 OtcR directly activates the transcription from oxy promoters in vivo. A. An illustration of the reporter plasmids. B. Detection of the regulatory relationship of OtcR and oxy promoters using gfp reporter plasmids in E.coli. C. Sequences of the direct repeats of native oxyl promoter (Poxyl) and two mutants (Poxyl1* and Poxyl2*). D. Detection of the interaction of OtcR with the direct repeat sequences of Poxyl, Poxy/1* and Poxyl2* using gfp reporters. The values are means \pm SD from three independent experiments.

was inserted into the reporter plasmids and OtcR was expressed, PoxyI $1^{*}$ showed less enhancement of fluorescence than that of PoxyI. Remarkably, the activity of Poxy 12* was no longer affected by OtcR (Figure 3D). These results indicated that OtcR lost the ability to interact with the mutant promoter Poxy $2 *$, thus had no effect on the transcription from Poxy $2^{*}$. Based on these results, we conclude that OtcR exerts its activator effect by binding to the $9 \mathrm{nt}$ direct repeats in PoxyI. This conclusion should also apply to the other promoters of $o x y$ cluster, as the consensus repeat sequences of these promoters share high degree of similarity.

\section{Overexpression of otcR}

Since otcR encodes a SARP cluster-situated regulator (CSR), positively regulating the expression of OTC biosynthesis, overexpression of $o t c R$ was adopted as a rational strategy to improve OTC production in S. rimosus M4018. An additional copy of otcR with its own promoter was cloned in plasmid pOtcR, the plasmid was transformed into M4018 to create M4018::otcR. OTC production of M4018::otcR increased significantly compared to that of M4018 (Figure 4A), both in R5 and FM media. To verify whether the improvement of OTC production was due to enhanced expression of oxy cluster, the activities of $o x y$ promoters were examined using GFP reporter in R5 medium. As shown in Figure 4B, green fluorescences conferred by the promoters of oxy were much higher in M4018::otcR than those in M4018, indicating that the overexpression of otcR indeed improved the transcription level of oxy genes. Thus, the overexpression of $o t c R$ is a viable strategy to improve OTC production in 4018.

\section{Improvement of OTC production by manipulating the expression levels of otcR}

As shown above, overexpression of otcR is an effective strategy to increase OTC production. To detect the optimum expression level of otcR, we designed new expression plasmids to manipulate the expression levels of $o t c R$. In a first attempt, otcR was cloned in an integrative vector under the control of a strong constitutive promoter SF14 [24], and the plasmid was transformed and integrated into the chromosome of S. rimosus M4018 to create M4018::SFotcR. The OTC production of M4018:: SFotcR was further increased in comparison with that of M4018::otcR, which contains one integrated copy of otcR under the control of its own promoter (Figure 5). Again, 


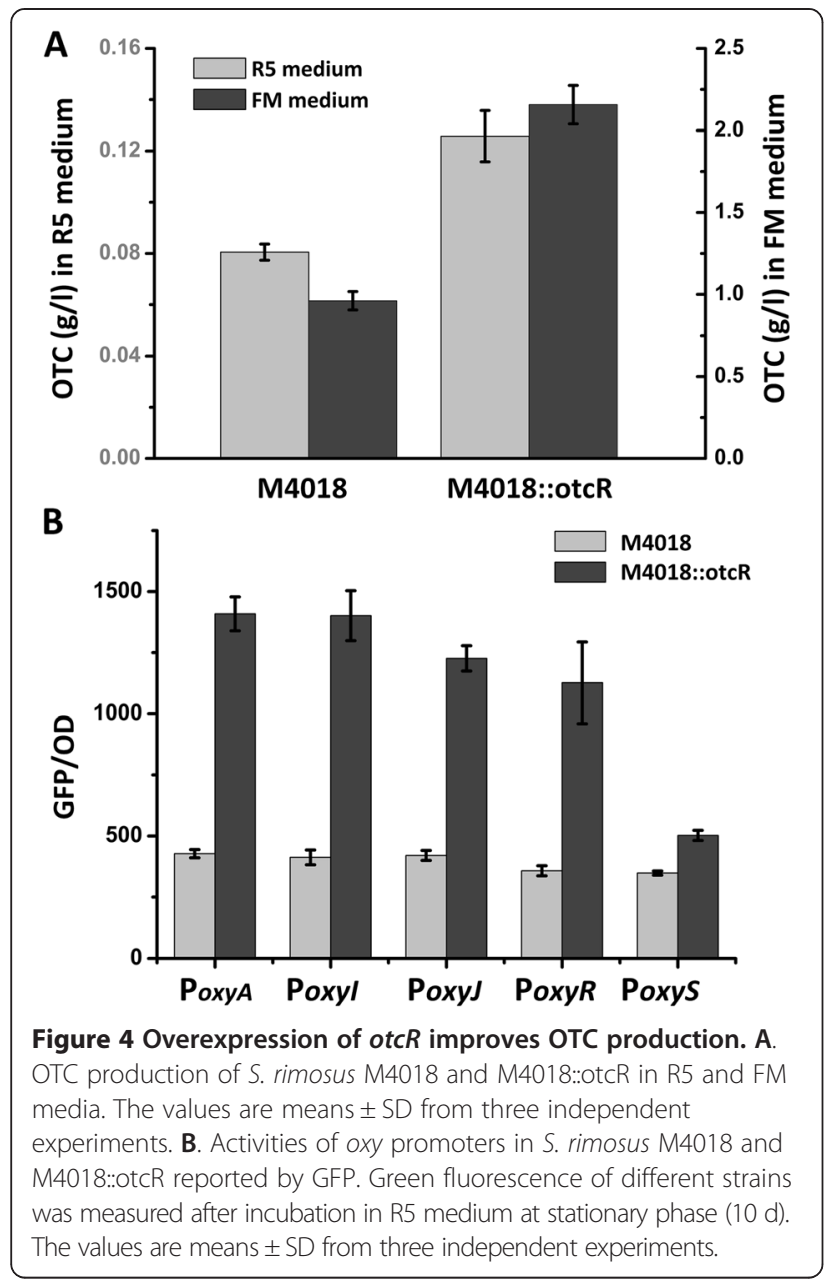

the activities of SF14 and otcR promoter were quantified by using GFP reporter, indicating that SF14 promoter had stronger transcriptional activity than $o t c R$ promoter at stationary phase (Additional file 3: Figure S3). These results demonstrated that the expression level of otcR is positively correlated with OTC production. To further improve OTC production, plasmids containing two or three copies of otcR driven by SF14 promoter were constructed and transformed into 4018 thus creating strains M4018::2SFotcR and M4018::3SFotcR, respectively. As shown in Figure 5, M4018::2SFotcR gave the highest OTC production $(6.24 \mathrm{~g} / \mathrm{l})$, which is 6.49 times as much as the production of M4018. While the OTC production of M4018::3SFotcR was less than in M4018::2SFotcR and M4018::SFotcR, indicating that the excessive expression of oxy genes does not coordinate the physiological condition of the producer.

\section{Discussion}

Productivity improvement of industrially used microorganisms is important to maintain the commercial viability of the corresponding industry. Previously, random

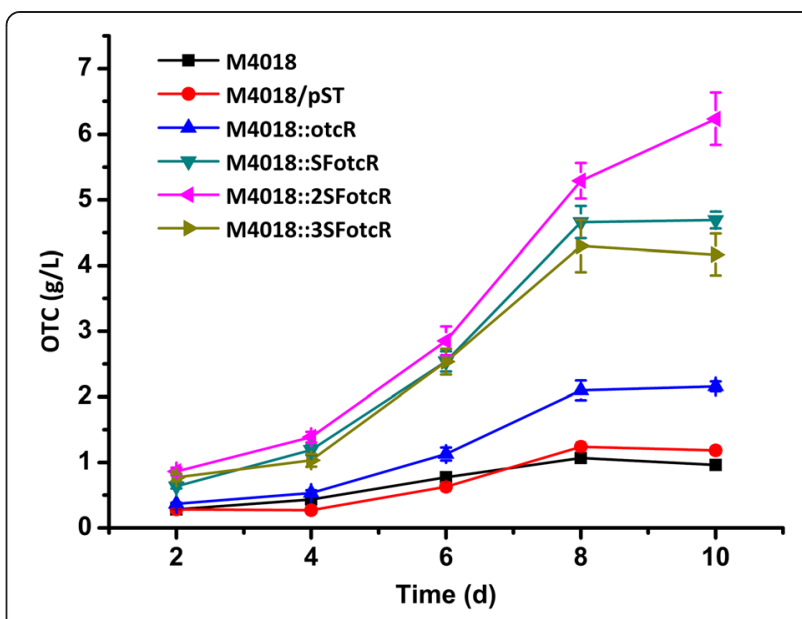

Figure 5 OTC production profiles of the engineered otcR overexpression strains. M4018/pST contains the control plasmid integrated in the genome of M4018; M4018::otcR contains an integrated copy of otcR driven by its own promoter; M4018::SFotcR, M4018::2SFotcR and M4018::3SFotcR contain one, two or three integrated copies of otcR driven by SF14 promoters. The values are means \pm SD from three independent experiments.

mutagenesis in combination with optimization of largescale industrial fermentations $[25,26]$ and rational metabolic engineering [27-29] have been carried out to improve OTC production in S. rimosus. However, due to lack of knowledge about the regulation of OTC biosynthesis, manipulations of regulatory genes to improve OTC production were not reported. Lesnik et al. [30] have identified a LAL (LuxR) family of transcriptional regulator OtcG, which located downstream of the otrA gene. OtcG plays a 'conditionally positive' role in OTC biosynthesis: inactivation of the otcG gene reduced the production of OTC by more than $40 \%$, but overexpression of otcG by introducing a second copy under the constitutive promoter ermE did not yield any statistically significant change in OTC production [30]. In this work, a SARP activator, OtcR, was identified in the oxy cluster. When we searched for otcR in the released genome of $S$. rimosus ATCC 10970 (accession no. ANSJ00000000), a single-base pair insertion mutation was found in the coding sequence of $o t c R$. The incomplete sequence (ELQ83266.1) was annotated as SARP family transcriptional regulator as well, which may be the main obstacle of dissecting the regulatory role of OtcR.

SARP regulators are characterized by their DNA-binding domains that resemble that of OmpR, and by an accompanying BTAD [31]. The sequence alignment of promoters regulated by SARPs revealed that the binding sites of these proteins contain common features [21], e.g. two direct repeats, the 3 ' repeat locates $8 \mathrm{bp}$ upstream of the -10 element (see Figure $2 \mathrm{~B}$ ), and the $5^{\prime}$ repeat overlaps with the -35 region of target promoter; the two repeats are separated from each other by $11 \mathrm{bp}$, corresponding to 
one complete turn of the DNA helix. Consequently, the two repeats of SARP-binding sites appear on the same face of the DNA helix, opposite to the face bound by RNA polymerase [21]. This topology would allow SARPs and RNA polymerase to have simultaneous access to the promoter region [21]. Therefore, to identify the existence of SARP-binding sites, such criteria were applied on the predicted promoter regions of oxy and ctc clusters. All the promoter regions of oxy showed the typical features of SARP-binding site.

SARP regulators, as activators of antibiotic biosynthesis in Streptomyces, are often found in the corresponding gene clusters, for example, ActII-ORF4 [32] and RedD [33] were identified in the actinorhodin and undecylprodigiosin gene clusters in S. coelicolor A3(2), respectively. Other examples include DnrI from the daunorubicin biosynthetic cluster in Streptomyces peucetius [34]; MtmR from the mithramycin biosynthetic cluster in Streptomyces argillaceus [35] and CcaR from the cephamycin and clavulanic acid super cluster in Streptomyces clavuligerus [36]. Therefore, to search for a SARPlike activator for OTC biosynthesis, the vicinity regions of oxy cluster were firstly investigated.

SARP regulators show great diversity in domain organization. The diversity is exemplified by their lengths, which vary from about 300 residues (e.g. ActII-ORF4 and DnrI $[32,34])$ to 1000 residues (e.g. AfsR, PimR and SanG $[21,37,38])$. The small SARP proteins usually contain two domains: an N-terminal DNA-binding domain and a Cterminal BTAD. In the cases of large SARPs, an extra ATPase domain and a TPR domain are extended downstream of the BTAD domain, which are involved in sensing endogenous signals (such as ADP/ATP pool) and regulating the activity of SARPs [20,21]. Compared to known SARPs, the length of OtcR is relatively short (251 amino acids), containing only the DNA binding and BTAD domains. Thus we deduce that OtcR is a pathway specific activator of OTC cluster not modulated by other signals. In addition, we were able to demonstrate that OtcR directly activates the expression of five oxy promoters in vivo and showed that the regulation is achieved by direct interaction of OtcR with the repeat sequence of oxy promoters to enhance their expression (Figure 3). These findings gave us confidence to rationally engineer otcR expression to improve OTC production in $S$. rimosus.

It is important to mention that the $9 \mathrm{nt}$ direct repeats of $o x y$ promoters encompass the 6 nt repeats identified from both oxy and ctc cluster, which could explain the finding that Ctc11 could activate the transcription of oxy in a heterologous host [13]. Based on sequence analyses, Ctc11 (CtcB) and OtcR share 46\% identities, in addition, the predicted SARP-binding sites were also identified in ctc cluster. Hence, Ctc11 is most likely the activator of ctc cluster and an ideal target for the engineering of CTC production.

SARP regulators as activators of antibiotic production have been targeted for engineering to improve the production of corresponding antibiotics in Streptomyces [16]. However, simple overexpression of activator genes may not give the best performance, fine-tuning of the expression levels of SARP activator should be tried to coordinate the expression of biosynthetic pathway with the physiology of producers. Recently, Sohoni et al. [18] used a synthetic promoter library to optimize the expression of ActII-ORF4; they discovered that a promoter giving the highest actinorhodin production level has a unique expression profile in term of strength and timing. In this work, otcR was overexpressed by the native and SF14 promoters, respectively. Our results showed that SF14 is stronger than the native otcR promoter at stationary phase (Additional file 3: Figure S3). As a consequence, the OTC production of M4018::SFotcR was significantly higher than that of M4018::otcR (Figure 5), suggesting higher expression levels of otcR are needed to further improve OTC production in S. rimosus M4018. So overexpression of otcR as tandem copies using SF14 promoter was tested, and further enhancement of OTC production level was observed. However, integrating only two copies of overexpressed otcR (M4018::2SFotcR) was found to confer the highest OTC production to M4018, three copies of otcR may lead to excessive burden on the cell, thus reducing OTC production. These results further emphasize the need to stepwise calibrate the expression level to determine the optimum expression level of activator genes.

\section{Conclusions}

In this work, OtcR was identified as a cluster-situated pathway specific activator of OTC biosynthesis in $S$. rimosus M4018. Moreover, manipulation of the expression levels of otcR could increase OTC production to more than six times in S. rimosus M4018. Therefore, engineering the expression of otcR is a promising alternative strategy for the engineering of OTC production strains.

\section{Methods}

\section{Strains, plasmids and culture conditions}

Strains and plasmids used in this study are listed in Table 1. E. coli TOP10 and DH5 $\alpha$ were used for cloning and GFP assay, respectively; E. coli ET12567/pUZ8002 was used for conjugation between $E$. coli and $S$. rimosus M4018. Streptomyces strains were grown on TSB medium (Oxoid) for genomic DNA isolation. Spores of S. rimosus were obtained on MS medium [39] after incubation at $30^{\circ} \mathrm{C}$ for 5 days. The seed cultures of $S$. rimosus strains for fermentation were obtained after growing in seed 
Table 1 Strains and plasmids used in this study

\begin{tabular}{|c|c|c|}
\hline Name & Description & Sources \\
\hline \multicolumn{3}{|l|}{ S. rimosus } \\
\hline M4018 & a strain used for commercial production of OTC & {$[27]$} \\
\hline$\Delta o t c R$ & otcR gene disruption mutant, derived from M4018 & This study \\
\hline$\Delta$ otcR::otcR & otcR complemented strain, derived from $\Delta \mathrm{otc} R$ & This study \\
\hline M4018::otcR & otcR overexpressed strain, derived from M4018 & This study \\
\hline M4018/pST & Integrated a control plasmid pST in the genome of M4018 & This study \\
\hline M4018::SFotcR & Integrated a copy of SF14-driven otcR in the genome of M4018 & This study \\
\hline M4018::2SFotcR & Integrated two copies of SF14-driven otcR in the genome of M4018 & This study \\
\hline M4018::3SFotcR & Integrated three copies of SF14-driven otcR in the genome of M4018 & This study \\
\hline \multicolumn{3}{|l|}{ E.coli } \\
\hline TOP10 & General cloning host for plasmid manipulation & Novagen \\
\hline ET12567 (pUZ8002) & Donor strain for conjugation between E. coli and Streptomycetes & [38] \\
\hline $\mathrm{DH} 5 \mathrm{a}$ & Host for reporter system & Novagen \\
\hline \multicolumn{3}{|l|}{ Plasmids } \\
\hline p4-16 h-7 s & Fosmid harbouring entire oxy gene cluster & This study \\
\hline pOtcRDM & For deletion otcR gene, containing left and right arms of otc $R$ & This study \\
\hline pGusT-SF14 & Derived from pSET152, containing SF14-driven gusA & [39] \\
\hline pST & Derived from pGusT-SF14, deleting the SF14-driven gusA in pGusT-SF14 & This study \\
\hline pSF14-otcR & Derived from pGusT-SF14, SF14-driven otcR & This study \\
\hline pSF142-otcR & Derived from pSF14-otcR, containing two copies of SF14-driven otcR & This study \\
\hline pSF143-otcR & Derived from pSF142-otcR, containing three copies of SF14-driven otcR & This study \\
\hline pOtcR & Derived from pGusT-SF14, containing a copy of otcR driven by its own promoter & This study \\
\hline pTAC & Template plasmid containing gfp & [40] \\
\hline pSF14-GFP & Derived from pGusT-SF14, containing SF14-driven gfp & This study \\
\hline pAGFP & Derived from pSF14-GFP, inserting oxyA promoter into upstream of gfp & This study \\
\hline plGFP & Derived from pSF14-GFP, inserting oxyl promoter into upstream of gfp & This study \\
\hline $\mathrm{pl1*GFP}$ & Derived from plGFP, inserting oxy $11^{*}$ promoter into upstream of $g f p$ & This study \\
\hline $\mathrm{pl} 2^{*} \mathrm{GFP}$ & Derived from plGFP, inserting oxy $/ 2^{*}$ promoter into upstream of $g f p$ & This study \\
\hline pJGFP & Derived from pSF14-GFP, inserting oxyJ promoter into upstream of gfp & This study \\
\hline pRGFP & Derived from pSF14-GFP, inserting oxyR promoter into upstream of gfp & This study \\
\hline pSGFP & Derived from pSF14-GFP, inserting oxyS promoter into upstream of $g f p$ & This study \\
\hline pRAGFP & Derived from pAGFP, inserting otcR driven by SF14 & This study \\
\hline PRIGFP & Derived from plGFP, inserting otcR driven by SF14 & This study \\
\hline $\mathrm{pRI} 1{ }^{*} \mathrm{GFP}$ & Derived from pl1*GFP, inserting otcR driven by SF14 & This study \\
\hline $\mathrm{pRI} 2^{*} \mathrm{GFP}$ & Derived from pl $2^{*} \mathrm{GFP}$, inserting otc $R$ driven by SF14 & This study \\
\hline pRJGFP & Derived from pJGFP, inserting otcR driven by SF14 & This study \\
\hline pRRGFP & Derived from pRGFP, inserting otcR driven by SF14 & This study \\
\hline PRSGFP & Derived from pSGFP, inserting otc $R$ driven by SF14 & This study \\
\hline pOtcR-AGFP & Derived from pAGFP, inserting otcR driven by its own promoter & This study \\
\hline pOtcR-IGFP & Derived from plGFP, inserting otcR driven by its own promoter & This study \\
\hline pOtcR-JGFP & Derived from pJGFP, inserting otcR driven by its own promoter & This study \\
\hline pOtcR-RGFP & Derived from pRGFP, inserting otcR driven by its own promoter & This study \\
\hline pOtcR-SGFP & Derived from pSGFP, inserting otcR driven by its own promoter & This study \\
\hline
\end{tabular}

*The asterisk is used to distinguish the engineered promoter from the native promoter (Poxyl). 
medium (3\% corn starch, $0.3 \%$ soya bean, $0.5 \%$ calcium carbonate, $0.4 \%\left(\mathrm{NH}_{4}\right)_{2} \mathrm{SO}_{4}, 0.5 \% \mathrm{NaCl}, 0.015 \% \mathrm{KH}_{2} \mathrm{PO}_{4}$ and $0.4 \%$ corn steep liquor) at $30^{\circ} \mathrm{C}$ for $1 \mathrm{~d}$. For GFP assay, S. rimosus strains were grown on R5 medium [39]. For measurement of OTC production, both R5 medium and optimum FM medium (5\% corn starch, 2\% soya bean, $1.4 \%$ calcium carbonate, $1.4 \%\left(\mathrm{NH}_{4}\right)_{2} \mathrm{SO}_{4}$, $0.4 \% \mathrm{NaCl}, 0.01 \% \mathrm{KH}_{2} \mathrm{PO}_{4}, 0.4 \%$ corn steep liquor, $0.001 \% \mathrm{CoCl}_{2}$ and $0.1-0.2 \%$ amylase) were used, the fermentation cultures were grown at $30^{\circ} \mathrm{C}$ for $10 \mathrm{~d}$. E. coli strains were grown in Luria-Bertani (LB) containing apramycin $(50 \mu \mathrm{g} / \mathrm{ml})$, kanamycin $(25 \mu \mathrm{g} / \mathrm{ml})$ or chloramphenicol $(25 \mu \mathrm{g} / \mathrm{ml})$ when necessary. S. rimosus exconjugants were selected on MS medium plates containing $50 \mu \mathrm{g} / \mathrm{ml}$ nalidixic acid and $500 \mu \mathrm{g} / \mathrm{ml}$ apramycin. High-fidelity PCR was performed using Q5 DNA polymerase (NEB) to obtain DNA fragments used for plasmid construction.

\section{Identification of otcR from a fosmid library of S. rimosus}

The genomic DNA library of S. rimosus M4018 was constructed using the EpiCentre CopyControl Fosmid Library Production kit in the pCC1FOS vector, according to the manufacturer's instructions. Briefly, genomic DNA of S. rimosus M4018 was size-fractionated in a $0.5 \%$ low-melting point agarose gel, and DNA fragments in the 33-48 $\mathrm{kb}$ range were collected for library construction. The purified genomic DNA fragments were ligated into a linearized pCC1FOS vector. The ligation mixture was then packaged into lambda phages using packaging extracts, and subsequently introduced into $E$. coli EPI-300. These cells were then plated onto LB agar plates containing $12.5 \mu \mathrm{g} / \mathrm{ml}$ chloramphenicol, and grown overnight aerobically at $37^{\circ} \mathrm{C}$, then the E. coli colonies were picked and stocked in 384-well-plates. PCR was carried out to screen for clones harboring the oxy gene cluster using the primers otc-up-F/otc-up- $\mathrm{R}$ and otc-down-F/otc-down-R. The regions upstream and downstream of oxy cluster were sequenced by fosmid walking.

The sequence of a fosmid clone (p4-16 h-7 s, Table 1) containing the oxy gene cluster were analyzed using Frameplot 4.0 beta (http://nocardia.nih.go.jp/fp4/) and the functions of coding regions were predicted by BLASTp (http://blast.ncbi.nlm.nih.gov/Blast.cgi). A gene encoding a SARP-type activator was found in a region upstream of $o x y$ cluster and designated $o t c R$. The nucleotide sequence of otcR has been submitted to the GenBank (Accession number: KP035101).

\section{Construction of otcR mutant ( $\Delta$ otcR) and complementation mutant ( $\Delta$ otcR::otcR)}

To construct otcR disruption plasmid pOtcRDM, a $3146 \mathrm{bp}$ fragment upstream of otcR was amplified with primers OtcR-up-F2/OtcR-up-R2 (Additional file 4: Table S1); a 3130 bp fragment downstream of otcR was amplified with primers OtcR-down-F2/OtcR-down-R2 (Additional file 4: Table S1) from genomic DNA of $S$. rimosus $\mathrm{M} 4018$. The two fragments were trimmed by appropriate enzymes and inserted into HindIII and EcoRI digested pKC1132 [39] to obtain pOtcRDM. The recombinant plasmid was introduced into E. coli ET12567 (pUZ8002) and conjugated into S. rimosus M4018. The transformants were selected for lower apramycin resistance to get double-crossover mutant $(\Delta \mathrm{otcR})$ following standard procedures [39]. The correct isolation of $\Delta$ otcR was confirmed by PCR amplification using primers $\Delta$ OtcR-Test2-F/ $\Delta$ OtcR-Test2-R (Additional file 4: Table S1), in which M4018 gave 1462 bp band, whereas $\Delta$ otcR gave a 600 bp band. To construct the complementation plasmid pOtcR, a 1029 bp fragment containing both promoter and otcR was amplified from the genomic DNA of M4018 with primers PotcR-F/PotcR-R (Additional file 4: Table S1). The PCR products were double digested with $B g l \mathrm{II} / \mathrm{Xba \textrm {I }}$ and ligated into the corresponding sites of pGusT-SF14 [40] to generate plasmid pOtcR. The plasmid was transformed into $\Delta$ otcR by conjugation. The resulting complementation strain ( $\Delta$ otcR::otcR) containing an integrated copy of otcR was confirmed by PCR with primers TestF2/TestR2 (Additional file 4: Table S1).

\section{Demonstration of the influence of OtcR on oxy promoters using GFP reporter in E.coli}

To assemble reporter plasmids for $o x y$ promoters, pGusTSF14 [40] was digested with BamHI/XbaI, and the green fluorescence gene $(g f p)$ was amplified from pTAC [41] with primers Gfp-F/Gfp-R. The two fragments were joined into a covalently sealed molecule using the recombination method described by Gibson et al. [42] to generate pSF14-GFP. The promoters of oxyA, oxyI, $o x y J$, oxyR and oxyS were amplified with primers PoxyA-gfp-F/PoxyA-gfp-R, PoxyI-gfp-F/PoxyI-gfp- R, PoxyJ-gfp-F/Poxyl-gfp-R, PoxyR-gfp-F/PoxyR-gfp-R and PoxyS-gfp-F/PoxyS-gfp-R (Additional file 4: Table S1), respectively. The promoterless pSF14-GFP fragments were obtained by $B g l \mathrm{II} / \mathrm{BamHI}$ double digestion. Using the same assembly method, the promoters of oxyA, oxyI, oxyJ, oxyR and oxyS were jointed with the promoterless pSF14GFP fragments to create the reporter plasmids pAGFP, pIGFP, pJGFP, pRGFP and pSGFP, respectively. To evaluate the regulatory effect of otcR on oxy promoters, the SF14 promoter and otcR fragments were amplified from pGusT-SF14 and pOtcR with primers SF14-F/SF14-R and Gib-otcR-F1/Gib-otcR-R1 (Additional file 4: Table S1), respectively. The plasmids pAGFP, pIGFP, pJGFP, pRGFP and pSGFP were digested by NheI and assembled with the above two fragments to obtain the corresponding reporter plasmids pRAGFP, pRIJFP, pRJGFP, pRRGFP and pRSGFP, 
respectively, which contain both an otcR under the control of SF14 promoter and a $g f p$ controlled by oxy promoters, the two genes were placed in the opposite orientations (Figure 3A). These plasmids were transformed into DH5 $\alpha$ to detect green fluorescence (excitation at $485 \mathrm{~nm}$; emission at $510 \mathrm{~nm}$, Synergy H4 Multi-Mode Reader). All fluorescence values were normalized to growth rates $\left(\mathrm{OD}_{600}\right)$. Each value and error bar represents the average and standard deviation of three experimental replicates, respectively.

\section{Verification of the interaction between OtcR and the direct repeat of oxyl promoter}

The promoter of oxyI (PoxyI) was chosen as a representative to investigate the interaction between OtcR and the predicted SARPs-binding direct repeats. To replace PoxyI by PoxyI1* in pIGFP and pRIGFP, primers PoxyI1*F/PoxyI1*R were used to amplify the linear fragments using pIGFP and pRIGFP as a template, respectively. Then these two PCR products were self-ligated to create pI1*GFP and pRI1*GFP, respectively. Using the same strategy, primers PoxyI2*F/PoxyI2*R were applied to generate $\mathrm{pI} 2 \% \mathrm{GFP}$ and $\mathrm{pRI} 2 \% \mathrm{GFP}$ linear fragments and the fragments were self-ligated to obtain pI2*GFP and pRI2*GFP plasmids. These plasmids were transformed into $\mathrm{DH} 5 \alpha$ and green fluorescence was detected as above.

\section{Construction of otcR overexpression strain M4018::otcR}

The complementation plasmid pOtcR was transformed into M4018 by conjugation to generate M4018::otcR, in which pOtcR was integrated in the chromosome of M4018. The control plasmid pST was obtained by selfligation of a 5632 bp fragment of pGusT-SF14 after digestion by $\mathrm{BamHI}$ and $\mathrm{XbaI}$; pST was transformed into M4018 by conjugation, and integrated into the chromosome of M4018 to generate M4018/pST as control.

To test the activities of oxy promoters in M4018::otcR, a fragment containing both otcR and its native promoter was amplified with primers Gib-PotcR-F/Gib-PotcR-R (Additional file 4: Table S1) using pOtcR as template. The PCR product was inserted into the NheI site of pAGFP, pIJFP, pJGFP, pRGFP and pSGFP to generate plasmid pOtcR-AGFP, pOtcR-IGFP, pOtcR-JGFP, pOtcRRGFP and pOtcR-SGFP, respectively. These plasmids were transformed into M4018 to obtain the corresponding strains in which the plasmids were integrated into the chromosome of M4018, these strains could report the activities of oxy promoters in M4018::otcR (containing 2 copies of $o t c R$ ). As controls, pAGFP, pIJFP, pJGFP, pRGFP and pSGFP were also transformed into M4018, the integration of these plasmids into the chromosome of M4018 generated strains that could report the activities of oxy promoters in M4018 from its single native copy of otcR. For green fluorescence detection, $200 \mu \mathrm{l}$ stationary phase $(10 \mathrm{~d})$ fermentation cultures were assayed as above. All fluorescence values were normalized to the biomass determined by diphenylamine colorimetric method [43]. Each value and error bar represents the average and standard deviation of three experimental replicates, respectively.

\section{Fermentation and OTC detection}

A spore suspension $\left(1.0 \times 10^{8}\right)$ was inoculated into $40 \mathrm{ml}$ of seed medium and grown for $24 \mathrm{~h}$. Then, $4 \mathrm{ml}$ of the first seed culture was inoculated into $40 \mathrm{ml}$ of optimum FM medium or R5 medium. All fermentation cultures were grown at $250 \mathrm{rpm}, 30^{\circ} \mathrm{C}$ for 10 days.

To quantify the production of OTC, the fermentation culture samples were adjusted to pH1.5-2.0 with $9 \mathrm{M}$ $\mathrm{HCl}$, and $1 \mathrm{ml}$ cultures were centrifuged at 12,000 rpm for $10 \mathrm{~min}$. Then, the samples were subjected to HPLC analysis on a Shimadzu Prominence HPLC system with dual $\lambda U V$ detector and YMC polymer C18 column $(4.6 \times 250 \mathrm{~mm})$. Separation was performed at the following conditions: $60 \% \mathrm{H}_{2} \mathrm{O}, 10 \%$ methanol, $20 \%$ acetonitrile and $10 \%$ phosphoric acid $(2 \mathrm{mM})$ with a constant flow rate of $1 \mathrm{ml} / \mathrm{min}$. The corresponding peak areas detected at $350 \mathrm{~nm}$ were used to calculate the concentration of OTC.

\section{Overexpression of otcR at different levels in S. rimosus M4018}

Plasmids that will allow otcR to integrate into the chromosome of M4018 one to three copies under the control of constitutive promoter SF14 were constructed. To generate pSF14-otcR, promoterless otcR fragment was amplified with primers OtcR-F/OtcR-R (Additional file 4: Table S1) and inserted into BamHI/XbaI digested pGusT-SF14. To generate pSF142-otcR, pSF14-otcR was digested by $\mathrm{Xba \textrm {I }}$ and ligated with the fragment amplified using primers Gib-OtcR-F2/Gib-OtcR-R2 (Additional file 4: Table S1) from pSF14-otcR. pSF143-otcR was constructed similarly by inserting Gib-OtcR-F2/Gib-OtcR-R2 (Additional file 4: Table S1) amplified fragment into pSF142-otcR. All plasmids were transformed into M4018 and the plasmids were integrated into the chromosome of S. rimosus M4018.

\section{Additional files}

Additional file 1: Figure S1. Organization of oxy cluster in S. rimosus (A) and alignment of amino acid sequences between Ctc11 and OtcR (B).

Additional file 2: Figure S2. Growth of S. rimosus M4018, $\Delta$ otcR, $\Delta$ otcR::otcR and M4018::otcR in R5 medium. The values are means \pm SD from three independent experiments.

Additional file 3: Figure S3. Comparison of the activities between the otcR (PotcR) and SF14 promoter using GFP reporters. The values are means \pm SD from three independent experiments.

Additional file 4: Table S1. Primers used in this study. 


\section{Competing interests}

The authors declare that they have no competing interests.

\section{Authors' contributions}

S Y and W W carried out experiments, analyzed the primary data and drafted the manuscript. $X W$ and $X J$ participated in the fermentation analysis. $Y Z$ constructed the fosmid library. S L participated in the data analysis. $K Y$ and Y Z supervised the whole research work and revised the manuscript. All authors read and approved the final manuscript.

\section{Acknowledgements}

The authors appreciate the kind donation of S. rimosus M4018 from Prof. Meijin Guo (East China University of Science and Technology) and plasmid pGusT-SF14 from Prof. Beatrix Suess (Technical University Darmstadt). This work was supported by funding from Shengxue Dacheng Pharmaceutical Co., Ltd, the National Natural Science Foundation of China (Grant Nos. U1361203, 31400034 and 31130001), the Ministry of Science and Technology of China (Grant No. 2013CB734001) and the Fundamental Research Funds for the Central Universities of China University of Mining and Technology (Beijing) (No. 2010YH05).

\section{Author details}

'Department of Environmental and Biological Engineering, School of Chemical and Environmental Engineering, China University of Mining and Technology (Beijing), D11 Xueyuan Road, Haidian District, Beijing 100083, People's Republic of China. ${ }^{2}$ State Key Laboratory of Microbial Resources, Institute of Microbiology, Chinese Academy of Sciences, 1 Beichen West Road, Chaoyang District, Beijing 100101, People's Republic of China. ${ }^{3}$ Shengxue Dacheng Pharmaceutical Co., Ltd, 50 Shengxue Road, Shijiazhuang 051430 Hebei, People's Republic of China.

Received: 19 December 2014 Accepted: 25 March 2015 Published online: 02 April 2015

\section{References}

1. Nelson ML, Levy SB. The history of the tetracyclines. Ann N Y Acad Sci. 2011;1241:17-32

2. Butler MJ, Friend EJ, Hunter IS, Kaczmarek FS, Sugden DA, Warren M. Molecular cloning of resistance genes and architecture of a linked gene cluster involved in biosynthesis of oxytetracycline by Streptomyces rimosus. Mol Gen Genet. 1989;215:231-8.

3. Kim ES, Bibb MJ, Butler MJ, Hopwood DA, Sherman DH. Sequences of the oxytetracycline polyketide synthase-encoding otc genes from Streptomyces rimosus. Gene. 1994;141:141-2.

4. Petkovic H, Thamchaipenet A, Zhou LH, Hranueli D, Raspor P, Waterman PG, et al. Disruption of an aromatase/cyclase from the oxytetracycline gene cluster of Streptomyces rimosus results in production of novel polyketides with shorter chain lengths. J Biol Chem. 1999;274:32829-34.

5. Binnie C, Warren M, Butler MJ. Cloning and heterologous expression in Streptomyces lividans of Streptomyces rimosus genes involved in oxytetracycline biosynthesis. J Bacteriol. 1989;171:887-95.

6. Peric-Concha N, Borovicka B, Long PF, Hranueli D, Waterman PG, Hunter IS. Ablation of the otcC gene encoding a post-polyketide hydroxylase from the oxytetracyline biosynthetic pathway in Streptomyces rimosus results in novel polyketides with altered chain length. J Biol Chem. 2005;280:37455-60.

7. Zhang W, Ames BD, Tsai SC, Tang Y. Engineered biosynthesis of a novel amidated polyketide, using the malonamyl-specific initiation module from the oxytetracycline polyketide synthase. Appl Environ Microbiol. 2006:72:2573-80.

8. Wang P, Gao X, Chooi YH, Deng Z, Tang Y. Genetic characterization of enzymes involved in the priming steps of oxytetracycline biosynthesis in Streptomyces rimosus. Microbiology. 2011;157:2401-9.

9. Wang $P$, Zhang W, Zhan J, Tang Y. Identification of OxyE as an ancillary oxygenase during tetracycline biosynthesis. Chembiochem. 2009;10:1544-50.

10. Wang P, Bashiri G, Gao X, Sawaya MR, Tang Y. Uncovering the Enzymes that Catalyze the Final Steps in Oxytetracycline Biosynthesis. J Am Chem Soc. 2013;135:7138-41.

11. McMurry LM, Levy SB. Revised sequence of OtrB (tet347) tetracycline efflux protein from Streptomyces rimosus. Antimicrob Agents Chemother. 1998;42:3050.
12. McDowall KJ, Thamchaipenet A, Hunter IS. Phosphate control of oxytetracycline production by Streptomyces rimosus is at the level of transcription from promoters overlapped by tandem repeats similar to those of the DNA-binding sites of the OmpR family. J Bacteriol. 1999;181:3025-32.

13. Wang P, Kim W, Pickens LB, Gao X, Tang Y. Heterologous expression and manipulation of three tetracycline biosynthetic pathways. Angew Chem Int Ed Engl. 2012;51:11136-40.

14. Ryan MJ, Lotvin JA, Strathy N, Fantini SE. Clone of the biosynthetic pathway for chlortetracycline and tetracline formation and cosmids useful therein. US Patent. 1999;5:866-410.

15. Martin JF, Liras P. Engineering of regulatory cascades and networks controlling antibiotic biosynthesis in Streptomyces. Curr Opin Microbiol. 2010;13:263-73.

16. Chen $Y$, Wendt-Pienkowski E, Shen B. Identification and utility of FdmR1 as a Streptomyces antibiotic regulatory protein activator for fredericamycin production in Streptomyces griseus ATCC 49344 and heterologous hosts. J Bacteriol. 2008;190:5587-96.

17. Bruheim P, Sletta H, Bibb MJ, White J, Levine DW. High-yield actinorhodin production in fed-batch culture by a Streptomyces lividans strain overexpressing the pathway-specific activator gene actll-ORF4. J Ind Microbiol Biotechnol. 2002;28:103-11.

18. Sohoni SV, Fazio A, Workman CT, Mijakovic I, Lantz AE. Synthetic promoter library for modulation of actinorhodin production in Streptomyces coelicolor A3(2). PLoS One. 2014;9:e99701.

19. Dehli T, Solem C, Jensen PR. Tunable promoters in synthetic and systems biology. Subcell Biochem. 2012;64:181-201.

20. Li R, Liu G, Xie Z, He X, Chen W, Deng Z, et al. PolY, a transcriptional regulator with ATPase activity, directly activates transcription of polR in polyoxin biosynthesis in Streptomyces cacaoi. Mol Microbiol. 2010;75:349-64.

21. Tanaka A, Takano Y, Ohnishi Y, Horinouchi S. AfsR recruits RNA polymerase to the afs $S$ promoter: a model for transcriptional activation by SARPS. J Mol Biol. 2007;369:322-33.

22. Zhu T, Cheng X, Liu Y, Deng Z, You D. Deciphering and engineering of the final step halogenase for improved chlortetracycline biosynthesis in industrial Streptomyces aureofaciens. Metab Eng. 2013;19:69-78.

23. Bailey TL, Boden M, Buske FA, Frith M, Grant CE, Clementi L, et al. MEME SUITE: tools for motif discovery and searching. Nucleic Acids Res. 2009;37:W202-8.

24. Labes G, Bibb M, Wohlleben W. Isolation and characterization of a strong promoter element from the Streptomyces ghanaensis phage 119 using the gentamicin resistance gene (aacC1) of Tn 1696 as reporter. Microbiology. 1997;143(Pt 5):1503-12.

25. RHODES PM, WINSKILL N, FRIEND EJ, WARREN M. Biochemical and Genetic Characterization of Streptomyces rimosus Mutants Impaired in Oxytetracycline Biosynthesis. J Gen Microbiol. 1981;124:329-38.

26. Al-Jawadi M, Calam CT. Physiology of a wild strain and high yielding mutants of Streptomyces rimosus, producing oxytetracycline. Folia Microbiol (Praha). 1987;32:388-401.

27. Tang Z, Xiao C, Zhuang Y, Chu J, Zhang S, Herron PR, et al. Improved oxytetracycline production in Streptomyces rimosus M4018 by metabolic engineering of the G6PDH gene in the pentose phosphate pathway. Enzyme Microb Technol. 2011;49:17-24.

28. Yu L, Cao N, Wang L, Xiao C, Guo M, Chu J, et al. Oxytetracycline biosynthesis improvement in Streptomyces rimosus following duplication of minimal PKS genes. Enzyme Microb Technol. 2012;50:318-24.

29. Yu L, Yan X, Wang L, Chu J, Zhuang Y, Zhang S, et al. Molecular cloning and functional characterization of an ATP-binding cassette transporter OtrC from Streptomyces rimosus. BMC Biotechnol. 2012;12:52.

30. Lesnik UaG A, Magdevska V, Fujs S, Raspor P, Hunter I, Petkovic H. Regulatory elements in tetracycline-encoding gene clusters: the otcG gene positively regulates the production of oxytetracycline in Streptomyces rimosus. Food Technol Biotechnol. 2009;47:323-30.

31. Wietzorrek A, Bibb M. A novel family of proteins that regulates antibiotic production in streptomycetes appears to contain an OmpR-like DNA-binding fold. Mol Microbiol. 1997;25:1181-4.

32. Arias P, Fernandez-Moreno MA, Malpartida F. Characterization of the pathway-specific positive transcriptional regulator for actinorhodin biosynthesis in Streptomyces coelicolor A3(2) as a DNA-binding protein. J Bacteriol. 1999;181:6958-68.

33. Narva KE, Feitelson JS. Nucleotide sequence and transcriptional analysis of the redD locus of Streptomyces coelicolor A3(2). J Bacteriol. 1990;172:326-33. 
34. Sheldon PJ, Busarow SB, Hutchinson CR. Mapping the DNA-binding domain and target sequences of the Streptomyces peucetius daunorubicin biosynthesis regulatory protein, Dnrl. Mol Microbiol. 2002;44:449-60.

35. Lombo F, Brana AF, Mendez C, Salas JA. The mithramycin gene cluster of Streptomyces argillaceus contains a positive regulatory gene and two repeated DNA sequences that are located at both ends of the cluster. J Bacteriol. 1999;181:642-7.

36. Santamarta I, Rodriguez-Garcia A, Perez-Redondo R, Martin JF, Liras P. CcaR is an autoregulatory protein that binds to the $c c a R$ and $c e f D-c m c l$ promoters of the cephamycin C-clavulanic acid cluster in Streptomyces clavuligerus. J Bacteriol. 2002;184:3106-13.

37. Anton N, Mendes MV, Martin JF, Aparicio JF. Identification of PimR as a positive regulator of pimaricin biosynthesis in Streptomyces natalensis. J Bacteriol. 2004;186:2567-75.

38. Liu G, Tian Y, Yang H, Tan H. A pathway-specific transcriptional regulatory gene for nikkomycin biosynthesis in Streptomyces ansochromogenes that also influences colony development. Mol Microbiol. 2005;55:1855-66.

39. Kieser T, Bibb MJ, Buttner MJ, Chater KF, Hopwood DA. Practical Streptomyces Genetics. Norwich, UK: The John Innes Foundation; 2000.

40. Rudolph MM, Vockenhuber MP, Suess B. Synthetic riboswitches for the conditional control of gene expression in Streptomyces coelicolor. Microbiology. 2013;159:1416-22.

41. Lou C, Stanton B, Chen YJ, Munsky B, Voigt CA. Ribozyme-based insulator parts buffer synthetic circuits from genetic context. Nat Biotechnol. 2012;30:1137-42.

42. Gibson DG, Young L, Chuang RY, Venter JC, Hutchison 3rd CA, Smith HO Enzymatic assembly of DNA molecules up to several hundred kilobases. Nat Methods. 2009;6:343-5

43. Zhao Y, Xiang S, Dai X, Yang K. A simplified diphenylamine colorimetric method for growth quantification. Appl Microbiol Biotechnol. 2013;97:5069-77.

\section{Submit your next manuscript to BioMed Central and take full advantage of:}

- Convenient online submission

- Thorough peer review

- No space constraints or color figure charges

- Immediate publication on acceptance

- Inclusion in PubMed, CAS, Scopus and Google Scholar

- Research which is freely available for redistribution 